(2) Open Access Full Text Article

\title{
An unexpected complication of chronic myelomonocytic leukemia: severe renal failure due to malignant tubulo-interstitial cell infiltration
}

This article was published in the following Dove Press journal: International Journal of Nephrology and Renovascular Disease 21 December 2015

Number of times this article has been viewed

Jerrold Spapen'

Karel Fostier ${ }^{2}$

Hendrik De Raeve ${ }^{3}$

Peter Janssens ${ }^{4}$

Herbert Spapen ${ }^{5}$

'Department of Internal Medicine, Universitair Ziekenhuis Brussel, Vrije Universiteit Brussel, ${ }^{2}$ Department of Hematology, ${ }^{3}$ Department of Pathology, ${ }^{4}$ Department of Nephrology, Universitair Ziekenhuis Brussel, ${ }^{5}$ Department of Intensive Care Medicine, Universitair

Ziekenhuis Brussel, Vrije Universiteit Brussel, Brussels, Belgium
Correspondence: Jerrold Spapen Department of Internal Medicine, Universitair Ziekenhuis Brussel, Vrije Universiteit Brussel, Laarbeeklaan I0I, Jette, 1090 Brussels, Belgium

Tel +32477480486

Email jerrold.spapen@hotmail.com
Abstract: Acute renal failure may complicate the course of a hematologic malignancy but is a highly unusual finding in patients with chronic myelomonocytic leukemia. Kidney biopsy is rarely performed in this setting, and the pathologic substrate underlying kidney injury is not well identified. We present a case of a biopsy-proven acute tubulo-interstitial nephritis due to massive infiltration of neoplastic myelomonocytic cells. Since the leukemic process involving the kidney may respond favorably to treatment, a renal biopsy should be considered in any patient presenting with unexplained severe or evolving kidney disease.

Keywords: chronic myelomonocytic leukemia, acute tubulo-interstitial nephritis, kidney biopsy, treatment

\section{Introduction}

Renal failure may complicate the course of hematologic malignancies. Chronic myelomonocytic leukemia (CMML) is an uncommon and complex blood cancer that very rarely affects the kidney. We present a case of progressive CMML-associated renal failure caused by acute tubulo-interstitial nephritis (ATIN) due to infiltration of neoplastic myelomonocytic cells.

The Ethical Committee of The University Hospital Brussels approved the study, but does not require patient consent for case presentations.

\section{Case report}

A 76-year-old man was admitted with intermittent high fever, polyuria, heavy fatigue, and excessive nocturnal transpiration. In 1 month, he had lost $5 \mathrm{~kg}$ of weight. He suffered from arterial hypertension, hypercholesterolemia, and ischemic cardiomyopathy and took acetylsalicylic acid, bisoprolol, atorvastatin, and occasionally sildenafil. $\mathrm{He}$ denied recent contact with sick people, traveling to tropical regions, or use of nonsteroidal anti-inflammatory or illicit drugs. Except for two home-held canaries, he was not exposed to animals. Six months before admission, a routine blood test showed mild normocytic anemia (hemoglobin $11.7 \mathrm{~g} / \mathrm{dL}$ ) with normal ferritin levels. At that time, no further diagnostic work-up was performed.

Physical examination on admission was normal. Relevant blood results are given in Table 1. Microscopic urinary examination showed pyuria but no hematuria or protein loss. Chest X-ray was normal. Contrast-enhanced abdominal computed tomography scan revealed enlarged edematous kidneys with preserved corticomedullary differentiation. Subsequent transesophageal echocardiography excluded endocarditis. Intravenous fluid and antibiotics were initiated. 
Table I Laboratory data

\begin{tabular}{|c|c|c|c|c|}
\hline Blood test & Reference range & On admission & After I week & After 2 months \\
\hline Serum creatinine $(\mathrm{mg} / \mathrm{dL})$ & $0.66-1.25$ & 2.60 & 5.13 & 1.29 \\
\hline CRP (mg/L) & $<5$ & 113.3 & 142.9 & 22.7 \\
\hline $\mathrm{LDH}(\mathrm{U} / \mathrm{L})$ & $313-618$ & 628 & 439 & 406 \\
\hline Uric acid (mg/dL) & $3.5-8.5$ & 9.8 & 7.2 & 6.3 \\
\hline Hemoglobin $(g / d L)$ & $13-16.5$ & 10.4 & 7.9 & 10.0 \\
\hline White-cell count (per $\mathrm{mm}^{3}$ ) & $3,600-9,600$ & 21,400 & 14,300 & 14,300 \\
\hline \multicolumn{5}{|l|}{ Differential count (\%) } \\
\hline Neutrophils & $4 I-74$ & 51.5 & 87.4 & 78.4 \\
\hline Eosinophils & $0-6$ & 0 & 0 & 0 \\
\hline Basophils & $0-2$ & 0.5 & 0.8 & 0 \\
\hline Lymphocytes & $19-44$ & 5.5 & 5.9 & 8.1 \\
\hline Monocytes & $3-13$ & 41.5 & 3.4 & 9.0 \\
\hline Myelocytes & $<1$ & I & 2.5 & 4.5 \\
\hline Platelet count (per $\mathrm{mm}^{3}$ ) & $158,000-480,000$ & 101,000 & 106,000 & 81,000 \\
\hline
\end{tabular}

Abbreviations: CRP, C-reactive protein; LDH, lactate dehydrogenase.

Under this treatment, pyuria and inflammation persisted, and serum creatinine rose to $5.13 \mathrm{mg} / \mathrm{dL}$. Fever peaks up to $40^{\circ} \mathrm{C}$ were documented. Extensive additional screening for viral, bacterial, and parasitic disease was negative. Antinuclear antibodies, anti-neutrophilic cytoplasmatic antibodies, and cryoglobulins were not detected. Complement levels were normal. Serum and urine protein electrophoresis was consistent with a nonspecific acute phase response. BenceJones protein was not detected. Serum and urinary lysozyme levels were normal.

A bone marrow aspirate and trephine biopsy were performed which showed dysgranulopoiesis and a hypercellular marrow, particularly populated with mononuclear cells and their progenitors. The karyotype was normal. BCR-ABL1 fusion transcript and rearrangements of the platelet-derived growth factor receptors A and B were negative. The bone marrow contained $17.5 \%$ blasts which confirmed the presence of CMML-2.

In light of the patient's atypical disease presentation characterized by galloping clinical and renal deterioration, a kidney biopsy was performed. Light microscopic examination showed interstitial infiltration with monocytic and reactive lymphoid cells. Focal lymphocyte infiltration of the tubular epithelium was observed. This "tubulitis" was in part associated with degenerative tubular changes. Glomerular or (peri) vascular inflammation was not seen (Figure 1). Blast cells were not detected. Interstitial fibrosis was absent. Monocytes occasionally formed interstitial aggregates simulating microgranulomas (Figure 2). Immunofluorescence microscopy could not detect immune and complement deposits. Acidfast, Periodic Acid-Schiff, and Gomori methenamine silver staining remained negative.
Antibiotics were stopped, and treatment with high-dose steroids (methylprednisolone $1 \mathrm{mg} / \mathrm{kg}$ /day) and hydroxycarbamide $(500 \mathrm{mg} /$ day $)$ was initiated. Serum creatinine level subsequently decreased to $1.93 \mathrm{mg} / \mathrm{dL}$. The patient's clinical condition steadily improved and fever subsided. After 10 weeks of progressive dose de-escalation, methylprednisolone was withdrawn. Kidney function further improved. Leukocyte and platelet counts remained stable, but anemia persisted, necessitating repeated packed cell transfusions.

\section{Discussion}

CMML is a clonal hematopoietic stem cell disorder characterized by an absolute monocytosis $\left(>10^{9}\right.$ cells/L) and both myelodysplastic and myeloproliferative bone marrow

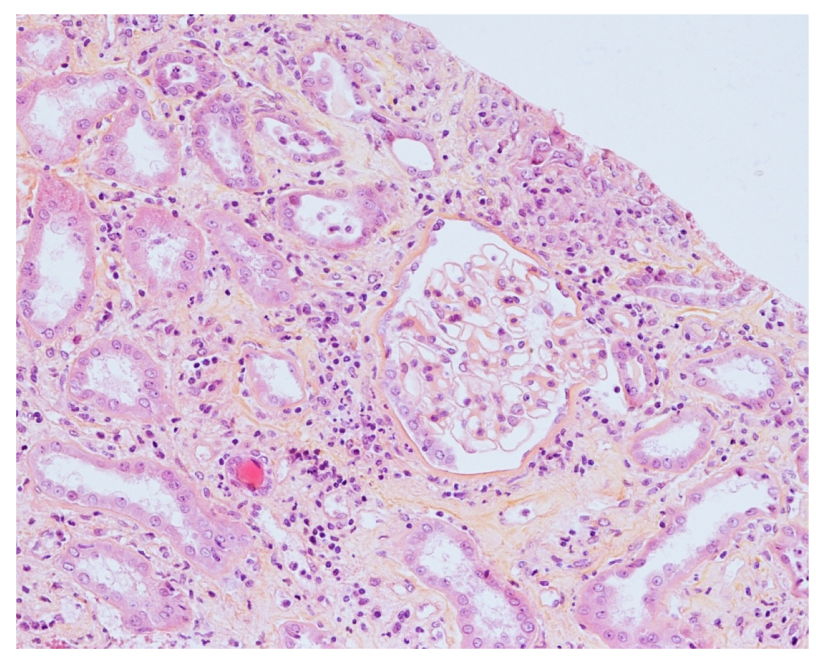

Figure I Kidney biopsy $(\times 200)$.

Notes: Hematoxylin and eosin staining showing interstitial infiltration with myeloid and monocytic cells. The glomeruli are morphologically normal. 


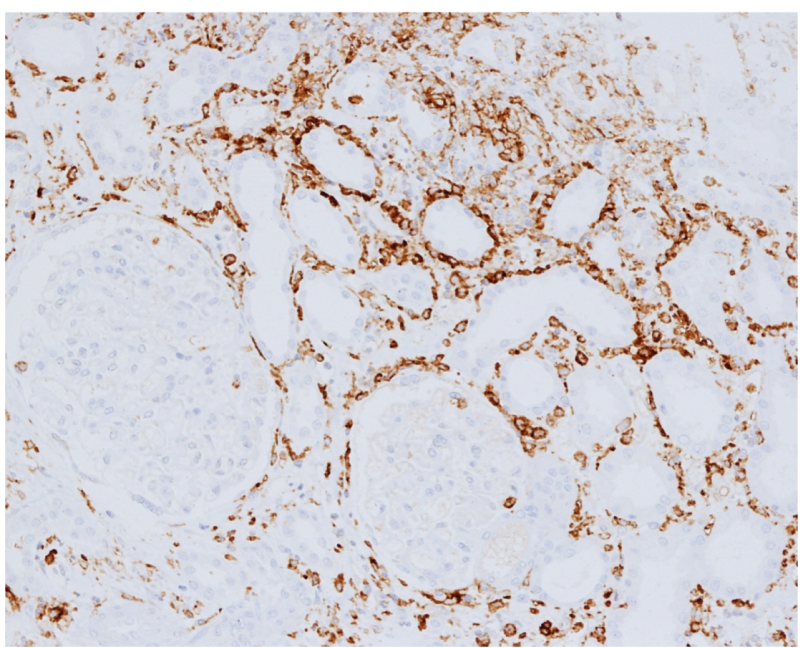

Figure 2 Kidney biopsy $(\times 200)$.

Notes: Immunohistochemical staining for CDI4 is positive in the numerous mature monocytes present in interstitium and around tubuli and glomeruli. Monocytes sometimes form aggregates simulating microgranulomas.

abnormalities. Disease onset is mostly insidious, and symptoms are highly variable. Patients may present with weight loss, fever, night sweats, and abdominal discomfort due to splenomegaly. Anemia is a common finding. Infectious and bleeding complications may occur. Differential diagnosis must account for a lot of conditions that are associated with monocytosis such as chronic infection (eg, infective endocarditis, tuberculosis, fungal and protozoal infections), connective tissue diseases (eg, systemic lupus erythematosus and sarcoidosis), and lipid storage disorders. ${ }^{1}$ As no single finding is pathognomonic of CMML, diagnosis is based on a combination of morphologic, histopathologic, and chromosomal abnormalities in bone marrow. Most patients have a hypercellular marrow with an expanded myelomonocytic compartment and features of dysplasia. Erythropoiesis is often decreased, and dysmegakaryopoiesis can be observed. Presence of the Philadelphia chromosome or the BCR-ABL1 fusion gene, as well as rearrangements of the platelet-derived growth factor receptors $\mathrm{A}$ and $\mathrm{B}$ must be excluded. Because the number of blasts (including myeloblasts, monoblasts, and promonocytes) is related to prognosis, the World Health Organization defined two different types of CMML: CMML-1 ( $<5 \%$ peripheral blasts and $<10 \%$ bone marrow blasts) and CMML-2 (5\%-19\% peripheral blasts and $10 \%-19 \%$ bone marrow blasts). Admittedly, it is challenging to discriminate monoblasts from mature monocytic cells. However, an experienced hematologist can make the difference based on cytomorphological characteristics. An additional argument in favor of chronic rather than acute leukemia may be the low $\mathrm{CD} 34^{+}$cell count $(0.2 \%)$ detected by bone marrow immunophenotyping. Acute leukemia ( $>19 \%$ blasts) develops in $15 \%-30 \%$ of cases. ${ }^{2}$

Kidney failure can complicate leukemia. Common causes include parenchymal infiltration by malignant cells, intrarenal leukostasis, tumor lysis syndrome, thrombotic microangiopathy, cryoglobulinemia, paraprotein deposition disease, chemotherapy-induced tubular or vascular toxicity, radiation injury, and ureteral obstruction from lymphadenopathy. ${ }^{3}$ Intrinsic renal involvement, though uncommon, is more likely to be encountered in CMML than in other forms of myelodysplasia. ${ }^{4}$ The scarce reports of biopsy-proven CMML-associated renal dysfunction mostly describe extracapillary or membranous glomerulonephritis and amyloid deposition. Proposed mechanisms underlying CMML-related glomerular impingement are abnormal antigen presentation, aberrant interactions between B- and T-cells, or production of high levels of circulating immune complexes or pro-inflammatory cytokines (eg, TNF-alpha) by activated monocytic cells. ${ }^{5}$ CMML-related acute kidney injury may also be caused by "blastic" infiltration of renal parenchyma and suburothelial connective tissue resulting in severe hematuria, vasculitis, and bilateral renal infarction. ${ }^{6}$ Myelomonocytic infiltration of the prostate and formation of bilateral reno-ureteral uric acid stones with consecutive "post"-renal failure have also been described. ${ }^{5}$ Finally, one case of acute renal failure secondary to tumor lysis after treatment with hydroxycarbamide and one case of tubular injury due to increased production and excretion of lysozyme were reported. ${ }^{3,7}$ The combination of interstitial inflammation, tubulitis, and degenerative tubular changes in the absence of vascular or glomerular damage confirmed the diagnosis of ATIN in our patient. Differential diagnosis of ATIN is extensive. Most common causes include allergic reactions (eg, drug-induced), infections, auto-immune and infiltrative processes (eg, lupus nephritis and sarcoidosis), inflammatory disease (eg, Sjögren's syndrome), and idiopathic conditions. Histologic features of ATIN are nonspecific. A marked interstitial infiltrate consisting primarily of mononuclear cells (T-lymphocytes and monocytes) without fibrosis is the hallmark of ATIN. Polymorphonuclear and eosinophil cells may also be detected. ${ }^{8}$ A marked monocytic interstitial infiltration was present in our patient. Even in the absence of formal proof of neoplastic infiltration (due to the absence of a reliable clonal marker), this infiltration was presumed leukemic by exclusion of other causes. Only two similar cases were previously published., ${ }^{4}$

Treatment of ATIN aims at controlling the underlying disease and/or eradicating the inciting agent. ${ }^{8}$ Since no 
randomized controlled trials comparing ATIN treatment have been conducted, the therapeutic approach should be individualized. Treatment of the hematologic malignancy may improve renal dysfunction induced by leukemic cell infiltration, but it remains unclear to what extent kidney function is resolved. Hydroxycarbamide remains the cornerstone of therapy for CMML patients with an elevated leukocyte count. The DNA-methylation inhibitors, azacitidine and decitabine, have been approved for treatment of patients with myelodysplastic syndromes. Several Phase II studies reported a good overall response of CMML to treatment with these hypomethylating agents. However, the retrospective and non-comparative nature of these trials precludes to draw definite conclusions. ${ }^{1}$ Allogeneic stem cell transplantation offers the best chance for long-term remission but remains controversial due to the lack of efficacy studies and the high comorbidity of an often elderly CMML population. ${ }^{1}$ Finally, comprehensive molecular profiling of CMML has identified a wide array of specific clonal mutations. This provides CMML with a unique genomic identity opening interesting diagnostic, therapeutic, and prognostic perspectives. ${ }^{10}$ Corticosteroids are commonly administered to patients with ATIN. Yet, outcome results are conflicting, and many issues remain unanswered (eg, mechanism of action) or topic of debate (eg, optimal dose and duration of therapy). ${ }^{11}$ Our patient responded well to a treatment combining high-dose steroids and hydroxycarbamide. Unless formally contraindicated (eg, infection-induced ATIN), steroid therapy should be considered in patients with ATIN whose kidney function fails to improve after attempting to control or eliminate the culprit disease or if vasculitis is evidenced.

In conclusion, ATIN secondary to myelomonocytic tubulo-interstitial infiltration should be included in the differential diagnosis of renal failure in CMML. If suspected, a renal biopsy must be considered to establish the diagnosis and to determine disease type and stage. Renal dysfunction is potentially reversible under treatment.

\section{Acknowledgment}

We are indebted to Kristin Jochmans, MD, PhD, Department of Laboratory Hematology, Universitair Ziekenhuis Brussel, Vrije Universiteit Brussel, Brussels, Belgium, for additional advice.

\section{Disclosure}

The authors report no conflicts of interest in this work.

\section{References}

1. Parikh SA, Tefferi A. Chronic myelomonocytic leukemia: 2013 update on diagnosis, risk stratification and management. Am J Hematol. 2013;88:968-974

2. Vardiman JW, Thiele J, Arber DA, et al. The 2008 revision of the World Health Organisation (WHO) classification of myeloid neoplasms and acute leukemia: rationale and important changes. Blood. 2009;114: 937-951.

3. Patel TV, Rennke HG, Sloan JM, DeAngelo DJ, Charytan DM. A forgotten cause of kidney injury in chronic myelomonocytic leukemia. Am J Kidney Dis. 2009;54:159-164.

4. Robinson GT, Sundaram KR, Dilly SA, Bevan DH, Andrews PA. Renal failure in a patient with chronic myelomonocytic leukemia. Nephrol Dial Transplant. 1997;12:1500-1502.

5. Enriquez R, Sirvent AE, Marin F, Pérez M, Alpera MR, Amorós F. Severe renal complications in chronic myelomonocytic leukemia. J Nephrol. 2008;21:609-614.

6. Hyams ES, Gupta R, Melamed J, Taneja SS, Shah O. Renal involvement by chronic myelomonocytic leukemia requiring nephroureterectomy. Rev Urol. 2009;11:33-37.

7. Otrock ZK, Taher AT, Mahfouz RA, Makarem JA, Shamseddine AI. Acute tumor lysis syndrome secondary to hydroxycarbamide in chronic myelomonocytic leukemia. Am J Hematol. 2006;81:220-221.

8. Praga M, González E. Acute interstitial nephritis. Kidney Int. 2010;77: 956-961.

9. Kobayashi K, Yokote T, Tsuji M, Takubo T, Inoue T, Hanafusa T. Renal infiltration associated with chronic myelomonocytic leukaemia. Br J Haematol. 2009; 147:414.

10. Padron E, Abdel-Wahab O. Importance of genetics in the clinical management of chronic myelomonocytic leukemia. J Clin Oncol. 2013;31: 2374-2376.

11. Praga M, Sevillano A, Auñón P, Gonzáles E. Changes in the aetiology, clinical presentation and management of acute interstitial nephritis, an increasingly common cause of acute kidney injury. Nephrol Dial Transplant. 2015;30:1472-1479.

\section{Publish your work in this journal}

The International Journal of Nephrology and Renovascular Disease is an international, peer-reviewed open-access journal focusing on the pathophysiology of the kidney and vascular supply. Epidemiology, screening, diagnosis, and treatment interventions are covered as well as basic science, biochemical and immunological studies. The journal welcomes

\section{Dovepress}

original research, clinical studies, reviews \& evaluations, expert opinion and commentary, case reports and extended reports. The manuscript management system is completely online and includes a very quick and fair peerreview system, which is all easy to use. Visit http://www.dovepress.com/ testimonials.php to read real quotes from published authors. 